\title{
Rebuilding sources of linear tracers after atmospheric concentration measurements
}

\author{
J.-P. Issartel \\ Ecole Nationale des Ponts et Chaussées, Centre d'Enseignement et de Recherche en Environnement Atmosphérique, France
}

Received: 11 March 2003 - Published in Atmos. Chem. Phys. Discuss.: 19 June 2003

Revised: 28 October 2003 - Accepted: 18 November 2003 - Published: 2 December 2003

\begin{abstract}
The identification of widespread sources of passive tracers out of atmospheric concentration measurements has become an important challenge of modern meteorology. The paper proposes some mathematical tracks to address the reconstruction of the complex space-time geometry of the sources of linear tracers. The methods are based upon the use of retroplumes. The inverse problem is addressed in a deterministic non statistical frame. The information obtained by local measurements is spread by introducing the concept of illumination. The constraint that the source is non negative is also addressed. The experimental source ETEX1 is rebuilt in order to evaluate an impulse response of the algorithms.
\end{abstract}

\section{Introduction}

We investigate the identification after concentration measurements of the source of a tracer scattered with a linear law. In a previous paper (Issartel and Baverel, 2003) we considered the localisation of a point source. This situation corresponded rather to accidental releases of pollutants (Baklanov, 1986; Sharan et al., 1995; Gallardo et al., 2002). An interpretation of the adjoint transport equation (Marchuk, 1992) as an inverse transport equation enabled to address an Eulerian version of backtracking leading to a cost-effective calculation of the inverse or adjoint plumes related to the measurements, and called "retroplumes". The same theoretical elements are used here to investigate another type of sources. Such tracers as carbon dioxide, carbon monoxide, methane, are emitted on wide areas. The source may vary in space and time with possibly negative sinks. From the now classical point of view of statistical inversions set up by Tarantola (1987) the present paper is primarily about the choice of the base functions used to interpret the measurements. The classical theory describes the use, not the choice, of such functions in the context of

Correspondence to: J.-P. Issartel

(issartel@cerea.enpc.fr) noisy measurements and model. The present development is compatible for an unaltered numerical price.

Sections 4 introducing the "eclairement" and 5 dealing with the constraint of positivity are the centre of this paper. To begin with in Sect. 2 the reader is reminded of an interpretation of the measurement operation as a scalar product. The consequence is developed in Sect. 3: the source of a measured linear tracer may be estimated by an orthogonal projection. This leads to a least square estimation meeting the classical problem of data assimilation: how irrigating the whole system with a rare local information? This question is addressed in Sect. 4 as the main point of this paper in terms of a deterministic property of the set of measurements, the "eclairement" or "illumination" describing which parts of the system are well or badly seen or not seen at all. Another important point, independent of the previous one, is addressed in Sect. 5, the possible requirement that the sought source should be non negative. The stability of the estimation against noises and computational errors is investigated in Sect. 6. The methods are compared in Sects. 7 and 8 with the data collected during the first ETEX experiment. In Sect. 9 the proposed strategy is placed in the present context of inversions.

ETEX1 was a twelve hour point release (Brandt et al., 1997). Some people may consider it is not natural applying to ETEX1 methods devoted to widespread sources. Our purpose is not here to obtain an extensive information about the source known to be a point source. Rather, we want to determine, like Seibert (2001), the reliability of a method, and the resolution with which a source is seen in order to determine which smooth enough sources could be well seen. We want to obtain something like the impulse response of an algorithm and it is necessary to use such a point source as ETEX1 for that.

Sections. 2 and 3 bear ideas going throughout the paper. The other sections are chained logically but the mathematical details are totally different and disconnected. 


\section{Reminder about the measurement product}

We shall extensively use the interpretation of the concentration measurement operation as a scalar product. This point of view classically introduces the adjoint equations for the sensitivity of the detectors. It was developed with various wordings by Marchuk (1964); Pudykiewicz (1998); Enting (2000); Penenko and Baklanov (2001) with privileged applications to radioactive species (Wotawa et al., 2003). Uliasz and Pielke (1991) introduced furthermore in this adjoint context the idea of inverse transport: the air sampled by the detector has originated from somewhere. A presentation of inverse transport has been proposed independently of adjoint techniques by Hourdin and Issartel (2000). The adjoint and inverse ideas were later described in (Issartel and Baverel, 2003) as different though equivalent.

The unknown source is described throughout the atmosphere as a positive or negative rate of release $\sigma(\boldsymbol{x}, t)$ at position $x$ and time $t$ in unit amount of tracer per unit mass of ambient air and unit time. This source linearly generates a concentration $\chi(\boldsymbol{x}, t)$ in unit amount of tracer per unit mass of air (mass mixing ratio). The link between $\chi$ and $\sigma$ stemming from a linear transport equation complemented with adequate boundary conditions may be described by a linear operator: $\chi=\mathcal{L}(\sigma)$.

The available tracer measurements $\mu_{i}, i=1, . ., n$, correspond to the analysis of air samples taken at various positions and dates. Sampling functions $\pi_{i}(\boldsymbol{x}, t)$ describing where and when the samples were taken are introduced in such a way that the measurements read as:

$\mu_{i}=\int_{\Omega \times \mathrm{T}} \rho(\boldsymbol{x}, t) \chi(\boldsymbol{x}, t) \pi_{i}(\boldsymbol{x}, t) d \boldsymbol{x} d t$

The integration is over the atmosphere $\Omega$ and the time domain $\mathrm{T} ; \rho$ is the air density.

The unit of the $\pi_{i}$ is a delicate though simple matter, it depends on the unit given to the measurements. Fundamentally the $\mu_{i}$ are amounts of tracer and the $\pi_{i}$ are in inverse unit of time (in fact in unit mass of air sampled per unit mass of ambient air and per unit time). Nevertheless the measurements are generally presented as concentrations per unit mass of air or per unit volume. This means that the sampling functions are normalised in such a way that $\int \rho \pi_{i} d \boldsymbol{x} d t=1$ or $\int \pi_{i} d \boldsymbol{x} d t=1$ respectively, which implies a division by the total mass or volume of the air sample.

The measurement operates as a scalar product $\mu_{i}=\mu\left(\chi, \pi_{i}\right)$ of the tracer concentration and sampling functions. It is therefore possible to consider the adjoint operator $\mathcal{L}^{*}$ and the adjoint retroplumes $r_{i}=\mathcal{L}^{*}\left(\pi_{i}\right)$ and to rewrite the measurements $\mu\left(\mathcal{L}(\sigma), \pi_{i}\right)=\mu\left(\sigma, \mathcal{L}^{*}\left(\pi_{i}\right)\right)$ as:

$\mu_{i}=\int_{\Omega \times \mathrm{T}} \rho(\boldsymbol{x}, t) r_{i}(\boldsymbol{x}, t) \sigma(\boldsymbol{x}, t) d \boldsymbol{x} d t$

The adjoint calculation of the $r_{i}$ is easy. In the case of a tracer passively transported by the air, with possibly some linear decay, the $r_{i}$ may be interpreted as retroplumes. They describe the concentration among the ambient air of the air to be sampled, by the $\pi_{i}$, before it was sampled. In this paper the convention is that all detector functions are normalised with respect to the mass of the samples, $\int \rho \pi_{i} d \boldsymbol{x} d t=1$. The $r_{i}$ are accordingly unitless mixing ratios.

The interpretation of the adjoint transport as a backward transport (Wigner, 1945; Hürwitz, 1948; Soodak, 1948; Weinberg and Wigner, 1958; Lewins, 1965) requires adequate conventions for the system of units of the source, concentration, sampling function and for the analytic form of the measurement product. The form 1 is one of those respecting the symmetries of the diffusion intended here as tied to a time-symmetric turbulence not distinguishing between any of two opposite directions. In the case of passive advection-diffusion $\mathcal{L}$ and $\mathcal{L}^{*}$ stand for the following forward and backward equations to be complemented with boundary conditions. The equations have opposite winds $\boldsymbol{v}$ and $-\boldsymbol{v}$ but equal diffusion $\zeta$.

$$
\begin{aligned}
& \frac{\partial \chi}{\partial t}+\boldsymbol{v} \cdot \nabla \chi+\zeta(\chi)=\sigma \\
& -\frac{\partial r}{\partial t}-\boldsymbol{v} \cdot \nabla r+\zeta(r)=\pi
\end{aligned}
$$

Diffusion is self-adjoint, $\zeta=\zeta^{*}$, because as the underlying turbulent motions are time symmetric, the adjoint-backward diffusion coincides with the forward diffusion. Smith (1956) already noticed this for a Fickian gradient diffusion.

The involvement of diffusion in backward transport is perplexing. As diffusion is irreversible, it seems to run counter the second principle. The obstacle is only apparent.

Firstly the backward integration is not intended at restoring the original position or geometry of the source. This is impossible since many sources could be an acceptable explanation for a single measurement. The backward integration just describes how the air in a sample was distributed in the atmosphere before being sampled. It is clear that far enough in the past this air was uniformly distributed.

Secondly, there is a creation of entropy towards the past by Eq. (4). This creation concerns the samples described by the $\pi_{i}$ which are not thermodynamically closed systems. Each sample is part of the atmosphere which is an open system that permanently refreshes its organisation. The entropy of the atmosphere as a whole is roughly constant. It is maintained by the degradation of solar into thermal radiation cooled from $5880 \mathrm{~K}$ down to $258 \mathrm{~K}$.

We stress that turbulent motions are not necessarily time symmetric. Convection for instance consists of rapid updrafts compensated by larger and slower downdrafts. Hence, the backward convection operator is obtained from the forward convection operator $\gamma=\gamma(\boldsymbol{x}, t)$ by means of a horizontal symmetry denoted $\gamma^{\dagger}$. Convection is accordingly subject to the constraint $\gamma^{*}=\gamma^{\dagger}$ (the constraints for the timesymmetric diffusion are stronger: $\left.\zeta=\zeta^{*}=\zeta^{\dagger}\right)$. 


\section{The starting idea}

This paragraph describes, to retrieve the sources, a starting idea based on a classical method of linear approximation introduced by Gram (1879); a more up to date presentation may be found in (Cheney, 1966; Marchuk, 1973). As we shall explain the idea is insufficient in our case. The insufficiency will be repaired in the next section.

The measurement product will be simply denoted $\mu(\phi, \psi)=(\phi, \psi)$ with an associated norm $\|\phi\|=\sqrt{(\phi, \phi)}$. The infinite dimensional vector space of finitely normed functions will be denoted $L^{2}(\Omega \times \mathrm{T})$.

The sought source $\sigma$ is subject to the following constraints making up an under-determined linear system:

$\mu_{i}=\int_{\Omega \times \mathrm{T}} \rho r_{i} \sigma(\boldsymbol{x}, t) d \boldsymbol{x} d t=\left(\sigma, r_{i}\right) \quad i=1, \ldots, n$

Measuring the $\mu_{i}$ amounts to determining the scalar product of the unknown source with the $r_{i}$. As a result we can determine the orthogonal projection $\sigma_{\|}$of $\sigma$ on the subspace the $r_{i}$ span in $L^{2}(\Omega \times \mathrm{T}) . \sigma_{\|}$has furthermore the property of being the least norm estimate of $\sigma$. The coefficients of the linear combination are obtained from the measurements by inverting the Gram covariance matrix $\mathbf{H}$ of the $r_{i}$ :

$\sigma_{\|}=\sum_{i=1}^{n} \lambda_{i} r_{i} \quad \boldsymbol{\mu}=\left[\begin{array}{c}\mu_{1} \\ : \\ \mu_{n}\end{array}\right] \quad \boldsymbol{\lambda}=\left[\begin{array}{c}\lambda_{1} \\ : \\ \lambda_{n}\end{array}\right]$

$\mathbf{H}=\left[h_{i, j}\right] \quad h_{i, j}=\left(r_{i}, r_{j}\right) \quad i=1, . ., n \quad j=1, . ., n$

$\boldsymbol{\mu}=\mathbf{H} \boldsymbol{\lambda} \quad$ or $\quad \boldsymbol{\lambda}=\mathbf{H}^{-1} \boldsymbol{\mu}$

Physical reasons may prescribe that $\sigma$ is non-zero only on a sub-domain $\mathcal{A} \subset \Omega \times \mathrm{T}$. This information may be handled by substituting for the adjoint retroplumes $r_{i}$ adequate restrictions $\left.r_{i}\right|_{\mathcal{A}}$ in the definition and calculation of $\sigma_{\|}$and $\mathbf{H}$, $h_{i, j}=\left(\left.r_{i}\right|_{\mathcal{A}},\left.r_{j}\right|_{\mathcal{A}}\right)$. The ETEX source has been investigated here as a surface source with, instead of a four-dimensional domain $\Omega \times \mathrm{T}$ a three dimensional domain $\Sigma \times \mathrm{T}$. Besides the use of restrictions $\left.r_{i}\right|_{\Sigma \times \mathrm{T}}$, the four dimensional mass-time element $\rho(\boldsymbol{x}, t) d_{3} \boldsymbol{x} d t$ is replaced in Eq. 5 by the three dimensional surface-time element $d_{2} x d t$ (this means that $\Sigma$ comes from a layer of thickness $\delta z(\boldsymbol{x}, t)$ inversely proportional to $\rho(\boldsymbol{x}, t)$ with hence a constant mass density per unit area).

Now the problem is as follows. Each retroplume displays a sharp peak concentrated around the corresponding detector. When they are combined to estimate the source, these peaks, all at different positions, cannot compensate. This foreseeable bad behaviour is clear on Fig. 2. The sources rebuilt according to this section display important positive or negative values around the position, in space and time, of the detectors. From a strict mathematical point of view, the above constructions are not sound. The retroplumes are generally not modelled as elements of $L^{2}(\Omega \times \mathrm{T})$. For instance a point detector at the origin with no wind, constant density $\rho_{0}$ and Fickian eddy diffusivity $\kappa$, is tied to a normalised retroplume with (see formula 17.50 of Seinfeld and Pandis, 1998):

$r(l, t)=\frac{\exp \left(\frac{l^{2}}{4 \kappa t}\right)}{(-4 \pi \kappa t)^{\frac{3}{2}}} \quad l \geq 0 \quad t \leq 0$

$\int_{\tau}^{0} \int_{0}^{+\infty} \rho_{0} r(l, t)^{2} 4 \pi l^{2} d l d t=+\infty \quad-\infty<\tau<0$

Accordingly $\|r\|^{2}$ does not exist and the retroplume $r$ cannot enter the composition of any Gram covariance matrix. In a numerical model the steepness of the detector and of the retroplume is bounded by the size of the meshes and of the time step. The mathematical obstacle remains hidden by this uncontrolled spontaneous smoothing.

Let's introduce the notations 10, 12 and definition 11:

$\boldsymbol{r}(\boldsymbol{x}, t)=\left[\begin{array}{c}r_{1}(\boldsymbol{x}, t) \\ : \\ r_{n}(\boldsymbol{x}, t)\end{array}\right] \in \mathbb{R}_{+}^{n}$
$\boldsymbol{g}(\boldsymbol{x}, t)=\left[\begin{array}{c}g_{1}(\boldsymbol{x}, t) \\ : \\ g_{n}(\boldsymbol{x}, t)\end{array}\right]=\mathbf{H}^{-1} \boldsymbol{r} \in \mathbb{R}^{n}$

for $\boldsymbol{a} \in \mathbb{R}^{n}, \quad \boldsymbol{b} \in \mathbb{R}^{n} \quad \boldsymbol{a} \cdot \boldsymbol{b}=\sum_{i=1}^{n} a_{i} b_{i}$

The families of functions $\left\{r_{1}, \ldots, r_{n}\right\}$ and $\left\{g_{1}, . ., g_{n}\right\}$ are dual with the meaning that ( $\delta$ is Kronecker's symbol):

$\left(r_{i}, g_{j}\right)=\int_{\Omega \times \mathrm{T}} \rho r_{i} g_{j} d x d t=\delta_{i, j}$

We would write with these notations:

$\sigma_{\|}(\boldsymbol{x}, t)=\lambda \cdot \boldsymbol{r}(\boldsymbol{x}, t)=\boldsymbol{\mu} \cdot \boldsymbol{g}(\boldsymbol{x}, t)$

\section{The illumination by the network}

The failure of the strategy proposed in the previous section may be interpreted as due to an unequal attention paid by the monitoring network to its environment. The problem was recognised in terms of probabilities by Ashbaugh et al. (1985) as emphasised by Stohl (1998). Still in terms of probabilities Baklanov (2000) clearly states the requirement for a correction factor that would homogenise this unequal attention: the present paper is mainly motivated by this question. Too much attention is paid to the neighbourhood of the detectors, the regions far away are poorly seen. This idea may be put into the quantitative definition of a function $E(x, t)$, that we call "eclairement" in French or "illumination" in English. 
7 stations, 48 measurements

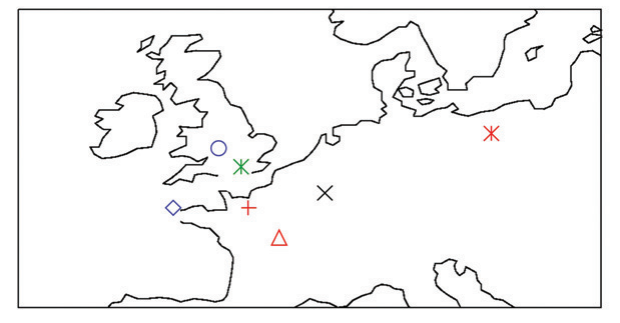

14 stations, 130 measurements

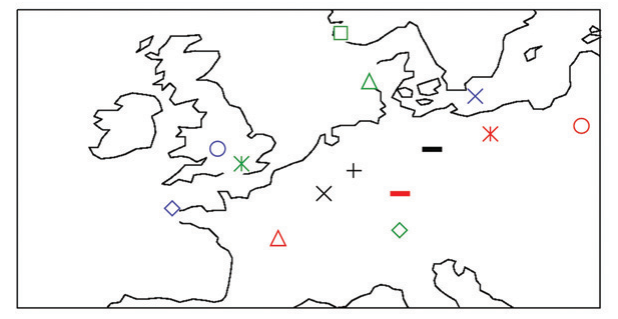

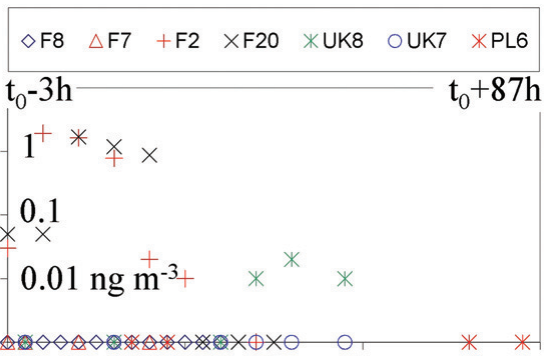

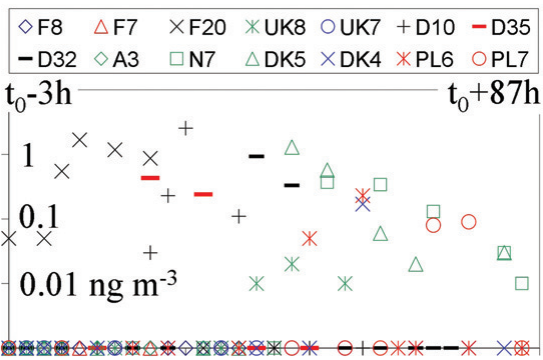

Fig. 1. Geographic and time distribution of the two selections of measurements used for the calculations. Upper two images, the 48 measurement selection, lower two images the 130 measurement selection. The time $t_{0}$ corresponds to the beginning of the pmch release in Monterfil, 23 October 1994, 16:00 UT; the first sampling period begins at $t_{0}-3 \mathrm{~h}$. Different detectors are indicated by different symbols. The values in $\mathrm{ng}$ of pmch per $\mathrm{m}^{3}$ are from the ETEX1 database.

The definition is given with tildes symbolising the precaution that only square summable approximations of the retroplumes are used, for instance the retroplumes produced by a numerical model. This ensures the existence of a Gram covariance matrix against the aforementioned obstacles:

$$
\begin{aligned}
\tilde{E}(\boldsymbol{x}, t) & ={ }^{t} \tilde{\boldsymbol{r}}(\boldsymbol{x}, t) \tilde{\mathbf{H}}^{-1} \tilde{\boldsymbol{r}}(\boldsymbol{x}, t) \\
& ={ }^{t} \tilde{\boldsymbol{g}}(\boldsymbol{x}, t) \cdot \tilde{\boldsymbol{r}}(\boldsymbol{x}, t) \geq 0
\end{aligned}
$$

The illumination is non negative because the matrix $\tilde{\mathbf{H}}^{-1}$ is positive definite. We obtain out of equations 13:

$\int_{\Omega \times \mathrm{T}} \rho \tilde{E}(\boldsymbol{x}, t) d \boldsymbol{x} d t=n$

Note that $\tilde{E}(\boldsymbol{x}, t)=0$ at a space-time position $(\boldsymbol{x}, t)$ only if no retroplume at all goes there. On the contrary $\tilde{\boldsymbol{r}}(\boldsymbol{x}, \mathrm{t})$ becomes very large in the neighbourhood of the detectors, $\tilde{E}(\boldsymbol{x}, t)$ should also become very large as the matrix $\tilde{\mathbf{H}}^{-1}$ in Eq. (15) is not position dependent. We think $\tilde{E}(\boldsymbol{x}, t)$ describes the share that goes to the neighbourhood of $(x, t)$ of the $n$ pieces of information produced by the measurements. Thus, $\tilde{E}$ would be an amount of information per unit mass of air (because of $\rho$ in Eq. 16) and per unit time.

These definition and interpretation are illustrated by Fig. $3 \mathrm{a}$ and $\mathrm{b}$ showing time integrated maps. Away from the monitoring system the illumination is weak and flat: sources there are out of its domain of relevance. The region of the detectors is covered by a spot of illumination with steep vari- ations. We consider as irrelevant these variations clearly followed by the sources estimated on Fig. 2. Their space scale is smaller than the distance between neighbouring detectors, no such detail of a source should be resolved.

In fact the illumination depends on the analytic form given to the measurement product. This property will be used to homogenise the share of the information. Let $f(\boldsymbol{x}, t)$ be a positive function, the equations $\mu_{i}=\left(\sigma, \tilde{r}_{i}\right)$ can be rewritten:

$$
\begin{gathered}
\mu_{i}=\int_{\Omega \times \mathrm{T}} \rho f r_{i}^{\prime} \sigma(\boldsymbol{x}, t) d \boldsymbol{x} d t \quad i=1, \ldots, n \\
r_{i}^{\prime}(\boldsymbol{x}, t)=\frac{\tilde{r}_{i}(\boldsymbol{x}, t)}{f(\boldsymbol{x}, t)}
\end{gathered}
$$

This transformation amounts to using a new scalar product $(\phi, \psi)^{\prime}$ (and norm $\|\psi\|^{\prime}$ ) to represent the measurement :

$$
\begin{aligned}
& (\phi, \psi)^{\prime}=\int_{\Omega \times \mathrm{T}} \rho f \phi(\boldsymbol{x}, t) \psi(\boldsymbol{x}, t) d \boldsymbol{x} d t \\
& \mu_{i}=\left(\sigma, r_{i}{ }^{\prime}\right)^{\prime} \quad i=1, \ldots, n
\end{aligned}
$$

For an adequate choice of $f$ the singularities or peaks of the retroplumes will be smoothed, the norms $\left\|r_{i}^{\prime}\right\|^{\prime}$ will be finite. The Gram covariance matrix $\mathbf{H}^{\prime}$ will be correctly defined leading to a sound estimate of the source:

$$
\begin{aligned}
& \mathbf{H}^{\prime}=\left[h_{i, j}^{\prime}\right] \quad h_{i, j}^{\prime}=\left(r_{i}{ }^{\prime}, r_{j}{ }^{\prime}\right)^{\prime}=\int_{\Omega \times \mathrm{T}} \rho \frac{\tilde{r}_{i} \tilde{r}_{j}}{f} d \boldsymbol{x} d t \\
& \lambda^{\prime}=\mathbf{H}^{\prime-1} \boldsymbol{\mu} \quad \sigma_{\|}^{\prime}(\boldsymbol{x}, t)=\lambda^{\prime} \cdot \boldsymbol{r}^{\prime}(\boldsymbol{x}, t)
\end{aligned}
$$



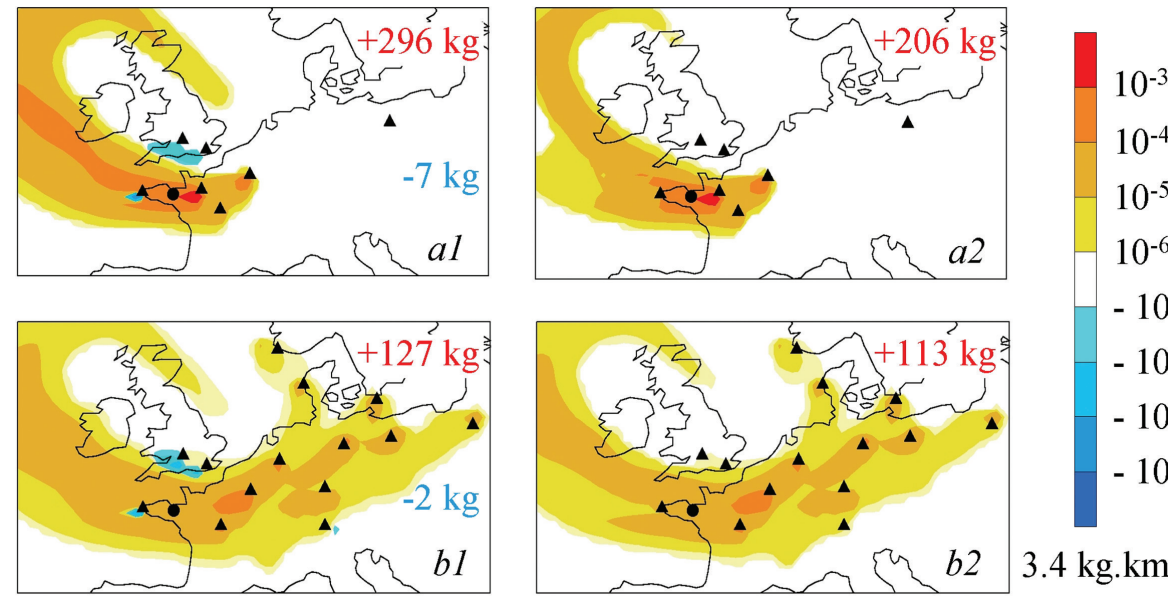

$-10^{-6}$

$-10^{-5}$

$-10^{-4}$

$-10^{-3}$

$3.4 \mathrm{~kg} \cdot \mathrm{km}^{-2}$

Fig. 2. Unsatisfactory results obtained out of a direct application of the orthogonal projection method for the two selections of 48 and 130 measurements in (a) and (b) respectively. The sources, evaluated as ground or sea level fluxes out of synthetic measurements, are integrated in time for each position. The positivity constraint is taken into account on the right column. The black dot indicates the position of Monterfil, the triangles indicate the position of the selected detectors.

Note that after smoothing an equation analogous to 16 may be written from which is inferred the smoothed share of information $E^{\prime}=f^{t} \boldsymbol{r}^{\prime} \mathbf{H}^{-1} \boldsymbol{r}^{\prime}$ :

$$
\begin{aligned}
& \int_{\Omega \times \mathrm{T}} \rho \tilde{E}^{t} \boldsymbol{r}^{\prime}(\boldsymbol{x}, t) \mathbf{H}^{\prime-1} \boldsymbol{r}^{\prime}(\boldsymbol{x}, t) d \boldsymbol{x} d t=n \\
& E^{\prime}(\boldsymbol{x}, t)=\frac{{ }^{t} \tilde{\boldsymbol{r}}(\boldsymbol{x}, t) \mathbf{H}^{\prime-1} \tilde{\boldsymbol{r}}(\boldsymbol{x}, t)}{f(\boldsymbol{x}, t)}
\end{aligned}
$$

The problem is now to choose a good $f$. There are two constraints. Firstly we would like the resulting $E^{\prime}$ to be as smooth as possible in order not to privilege any position; in particular the detector peaks of $\tilde{E}$ should be smoothed out. Secondly the regions badly seen and especially not seen at all should not be attributed an excessive importance. The competition between these constraints implies that to the set of measurements is attached a space time domain of relevance. Inside this domain the illumination $E^{\prime}$ should be as flat as possible, outside it should be as weak as possible.

The results of Sects. 7, 8 were obtained for the following choice of $f ; \tilde{E}_{\max }$ is the maximum value reached by $\tilde{E}$ :

$f(\boldsymbol{x}, t)=\max \left[\tilde{E}(\boldsymbol{x}, t), \frac{\tilde{E}_{\text {max }}}{1000}\right]$

This is sufficient to cancel the peaks of illumination rising from the background with this factor 1000 for horizontal dimensions of about $100 \mathrm{~km}$ (attenuated on Fig. 3a and $\mathrm{b}$ by the time integration) unreasonably small compared to the distances between neighbouring stations. Close to and away from the detectors the smoothed "éclairement" is respectively $E^{\prime}=\frac{{ }^{t} \tilde{\boldsymbol{r}} \mathbf{H}^{-1} \tilde{\boldsymbol{r}}}{{ }^{t} \tilde{\boldsymbol{r}}{ }^{-1} \tilde{\boldsymbol{r}}}$ or $E^{\prime}=1000 \frac{{ }^{t} \tilde{\boldsymbol{r}} \mathbf{H}^{\prime-1} \tilde{\boldsymbol{r}}}{\tilde{E}_{\max }}$. Close to the detectors $\|\tilde{r}\|$ is large but $E^{\prime}$ remains bounded. Away $\|\tilde{r}\|$ is small. Both above constraints are fulfilled as shown by Fig. 3a and $b$.

The calculation of $\sigma_{\|}^{\prime}$ requires the calculation and inversion of a first Gram covariance matrix $\tilde{\mathbf{H}}$ to obtain $\tilde{E}$, and of a second smoothed Gram covariance matrix $\mathbf{H}^{\prime}$ to finally obtain $\sigma_{\|}^{\prime}$. The price is the same as required classically for the calculation and inversion of one asymmetric measurement matrix (see Sect. 9). In our calculations the conditioning of $\mathbf{H}^{\prime}$ was always a bit less favourable than that of $\tilde{\mathbf{H}}$. Both never exceeded 60. As shown in Sect. 6, it is the square root of the conditioning that counts. Hence regularisation techniques are unessential at the present stage.

What happens when $\tilde{\boldsymbol{r}}$ tends to $\boldsymbol{r}$ ? Then, the illumination $E$ concentrates like Dirac masses at the position of the ideal point detectors. Our opinion is that the $r_{i}{ }^{\prime}=\frac{\tilde{r}_{i}}{f}$ and the estimate $\sigma_{\|}^{\prime}$ tend to physically relevant limits but we did not investigate this point deeply.

\section{The positivity constraint}

There are physical circumstances in which the source $\sigma(\boldsymbol{x}, t)$ is known to be non negative and should be attributed a non negative estimate. This will be the case when considering such tracers as methane, carbon monoxide, pesticides, etc. We recall that the linear decay or transformation processes should be considered separately in the equations but not in the source term. In fact we shall seek a non negative smooth estimate but the method developed in this section towards positivity and that presented in the previous one towards smoothness are totally independent. They can be combined without restriction. The presentation below is written with 

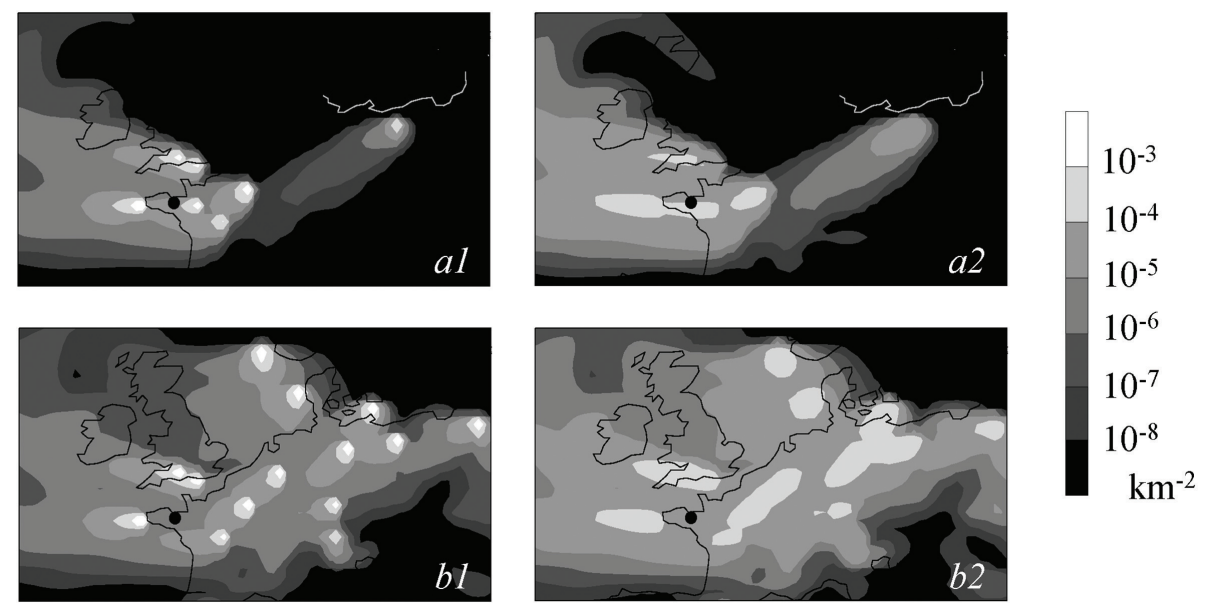

Fig. 3. Time integrated maps of "eclairement" or "illumination" for the selections of 48 measurements in (a) and 130 in (b): initial illumination on the left, smoothed illumination on the right. The illumination is a geometric property describing, independently of the effective values of the measurements, which regions have been observed. The black parts of the maps correspond to areas poorly or not seen at all. For the detectors these areas are as if they did not exist, sources there would have no influence on the measurements. The unit, $\mathrm{km}^{-2}$ after the time integration and for sources known to be surface sources, may be regarded as a number of information per unit area for an integrated total of 48 or 130 respectively.

a smoothing function $f$ and smoothed variables. The raw adjoint retroplumes could as well be used with $f \equiv 1$.

The linearly built estimate $\sigma_{\|}^{\prime}$ may be termed algebraic to mean that there is no reason why it should be everywhere non negative. It may be alternatively defined as the argument $s \in L^{2}(\Omega \times \mathrm{T})$ of the norm $\|s\|$ achieving its minimum value subject to constraints thus leading to an analogous definition of the least norm positive estimate $\sigma_{+}^{\prime}$ :

$\sigma_{\|}^{\prime}=\operatorname{argmin}\left\{\|s\|,\left(s, r_{i}^{\prime}\right)^{\prime}=\mu_{i}, i=1, . ., n\right\}$

$\sigma_{+}^{\prime}=\operatorname{argmin}\left\{\|s\|,\left(s, r_{i}^{\prime}\right)^{\prime}=\mu_{i}, i=1, . ., n, s \geq 0\right\}$

The non negative constraint can be taken into account in a computationally cost-effective way. The rest of the section is devoted to the mathematical details that may or may not interest the reader. We iteratively use the conjecture 24 to reach a positive estimate finally controlled to coincide with the definition 23 by means of an optimality criterion. Numerical results of de Villiers et al. (1999) suggest the following property (a conjecture as emphasised by the question mark):

$\sigma_{\|}(\boldsymbol{x}, t) \leq 0 \stackrel{?}{\Longrightarrow} \sigma_{+}^{\prime}(\boldsymbol{x}, t)=0, \quad(\boldsymbol{x}, t) \in \Omega \times \mathrm{T}$

First, starting from $\xi_{0}=\sigma_{\|}^{\prime}$, and iteratively through sources $\xi_{v}$ all constrained by the measurements, a positive estimate $\xi_{\infty}$ is obtained with no least norm requirement. The iterations are simple: each estimate $\xi_{v+1}$ is constrained to be zero wherever $\xi_{v}(\boldsymbol{x}, t)$ is negative or zero (there $\sigma_{+}^{\prime}$ is conjectured to vanish). The first iteration is given as an example.

The space time domain divides into two sub-domains, $\Omega \times \mathrm{T}=\mathcal{O}_{0}^{+} \cup \mathcal{O}_{0}^{-}$, where respectively $\xi_{0}(\boldsymbol{x}, t)>0$ and $\xi_{0}(\boldsymbol{x}, t) \leq 0$. As $\xi_{1}$ will vanish in $\mathcal{O}_{0}^{-}$the measurement constraints 17 become:

$\mu_{i}=\int_{\mathcal{O}_{0}^{+}} \rho f r_{i}{ }^{\prime} \xi_{1}(\boldsymbol{x}, t) d \boldsymbol{x} d t \quad i=1, \ldots, n$

Then $\xi_{1}$ is obtained by inverting a Gram covariance matrix $\mathbf{H}_{(1)}^{\prime}$ integrated on the smaller domain $\mathcal{O}_{0}^{+}$:

$$
\begin{array}{lr}
\mathbf{H}_{(1)}^{\prime}=\left[h_{(1) i, j}^{\prime}\right] & h_{(1) i, j}^{\prime}=\int_{\mathcal{O}_{0}^{+}} \rho f r_{i}^{\prime} r_{j}^{\prime} d \boldsymbol{x} d t \\
\boldsymbol{l}_{1}=\mathbf{H}_{(1)}^{\prime}{ }^{-1} \boldsymbol{\mu} & \xi_{1}(\boldsymbol{x}, t)= \begin{cases}\boldsymbol{l}_{1} \cdot \boldsymbol{r}^{\prime}(\boldsymbol{x}, t) & \text { in } \mathcal{O}_{0}^{+} \\
0 & \text { in } \mathcal{O}_{0}^{-}\end{cases}
\end{array}
$$

The positive domain iteratively shrinks until a limit $\mathcal{O}_{\infty}^{+}$, the negative one expands until $\mathcal{O}_{\infty}^{-}, \boldsymbol{l}_{v}$ accumulates at a limit $\boldsymbol{l}_{\infty}$. In our calculations the number of iterations required to reach $\xi_{\infty}$ was always about 10 , it never exceeded 20 :

$\xi_{\infty}(\boldsymbol{x}, t)=\left\{\begin{array}{lr}\boldsymbol{l}_{\infty} \cdot \boldsymbol{r}^{\prime}(\boldsymbol{x}, t)>0 & \text { in } \mathcal{O}_{\infty}^{+} \\ 0 & \text { in } \mathcal{O}_{\infty}^{-}\end{array}\right.$

The aim is now to check that the norm of $\xi_{\infty}$ is minimal among the positive estimates in order to conclude : $\xi_{\infty}=\sigma_{+}^{\prime}$. Due to the convexity of the norm and of the constraints the definitions 22 and 23 come under convex optimisation theory (Rockafellar, 1970). Hence the Karush, Kuhn and Tucker necessary optimality conditions become sufficient. The criterion is derived here from the Kuhn-Tucker Lagrangian function $\Lambda$ defined for sources $s \in L^{2}(\Omega \times \mathrm{T})$ and multipliers 
$a \in \mathbb{R}^{n}$ of the equality measurement constraints:

$$
\begin{aligned}
\Lambda(s, \boldsymbol{a}) & =(s, s)^{\prime}+\sum_{i=1}^{n} a_{i}\left(\left(s, r_{i}^{\prime}\right)^{\prime}-\mu_{i}\right) \\
& =\int_{\Omega \times \mathrm{T}} \rho f\left(s^{2}+\sum_{i=1}^{n} a_{i} s r_{i}^{\prime}\right) d \boldsymbol{x} d t-\boldsymbol{a} \cdot \boldsymbol{\mu}
\end{aligned}
$$

$\xi_{\infty}$ is optimal if there exist Lagrange multipliers $\boldsymbol{a}$ in such a way that $\partial_{s} \Lambda\left(\xi_{\infty}, \boldsymbol{a}\right) \geq 0$ and $\xi_{\infty} \partial_{s} \Lambda\left(\xi_{\infty}, \boldsymbol{a}\right)=0$ which means for each $(\boldsymbol{x}, t) \in \Omega \times \mathrm{T}$ (after eliminating $\rho f>0$ ):

$2 \xi_{\infty}(\boldsymbol{x}, t)+\boldsymbol{a} \cdot \boldsymbol{r}^{\prime}(\boldsymbol{x}, t) \geq 0$

$\xi_{\infty}(\boldsymbol{x}, t)\left[2 \xi_{\infty}(\boldsymbol{x}, t)+\boldsymbol{a} \cdot \boldsymbol{r}^{\prime}(\boldsymbol{x}, t)\right]=0$

In $\mathcal{O}_{\infty}^{+}$we have $\xi_{\infty}>0$ so that according to Eq. 30 necessarily: $\boldsymbol{a}=-2 \boldsymbol{l}_{\infty}$. Finally to decide the optimality of $\xi_{\infty}$ we look at Eq. 29 for $(\boldsymbol{x}, t) \in \mathcal{O}_{\infty}^{-}$:

$\xi_{\infty}=\sigma_{+}^{\prime} \Longleftrightarrow \boldsymbol{l}_{\infty} \cdot \boldsymbol{r}^{\prime}(\boldsymbol{x}, t) \leq 0$ in $\mathcal{O}_{\infty}^{-}$

The following inequality were obtained indicating that $\xi_{\infty}$ is at least a good approximation of $\sigma_{+}^{\prime}$. The left part is the amount of tracer positively emitted by $\boldsymbol{l}_{\infty} \cdot \boldsymbol{r}^{\prime}$ in $\mathcal{O}_{\infty}^{-}$in contravention of the optimality conditions 31 . The right part is the amount of tracer released by $\xi_{\infty}$ i.e. by $\boldsymbol{l}_{\infty} \cdot \boldsymbol{r}^{\prime}$ in $\mathcal{O}_{\infty}^{+}$.

$$
\begin{array}{r}
\int_{\mathcal{O}_{\infty}^{-}} \rho f \max \left[0, \boldsymbol{l}_{\infty} \cdot \boldsymbol{r}^{\prime}(\boldsymbol{x}, t)\right] d \boldsymbol{x} d t \leq \ldots \\
\ldots 310^{-2} \int_{\mathcal{O}_{\infty}^{+}} \rho f \boldsymbol{l}_{\infty} \cdot \boldsymbol{r}^{\prime}(\boldsymbol{x}, t) d \boldsymbol{x} d t
\end{array}
$$

We tried to improve the optimality of $\xi_{\infty}$ by relaxing the violated optimality conditions. This means the iterative process stopped at $\xi_{\infty}$ was relaunched by adding to the positive domain $\mathcal{O}_{+}^{\infty}$ the parts of $\mathcal{O}_{-}^{\infty}$ where the optimality conditions were violated or weakly satisfied. The inequality 32 could not be improved significantly.

We first suspected the conjecture 24 to be true in such quadratic optimal problems as 22, 23 with non negative coefficients $r_{i}^{\prime}$ and constraint parameters $\mu_{i}$. The following counter-example is due to Jean-Bernard Baillon (2003) who kindly communicated it to us. It implies 4-dimensional "retroplumes" $r_{1}=(2,5,4,4) \quad r_{2}=(1,6,9,4) \quad r_{3}=(7,8,3,3)$; the constraints for $s \in \mathbb{R}^{4}$ are $r_{i} \cdot s=1, i=1,2,3$. The orthogonal projection is $\sigma_{\|}=\left(-\frac{307}{23982}, \frac{904}{11991},-\frac{141}{7994}, \frac{308}{1713}\right)$, the positive optimum is $\sigma_{+}=\left(\frac{1}{39}, \frac{1}{39}, 0, \frac{8}{39}\right)$.

The above algorithm is interesting because it is cost effective and respects the logic of the physical problem which is important when regularising techniques have to be used. Rather than controlling and improving the optimality conditions at the end of the calculation, of course it would be more satisfactory understanding a possible link between the retroplumes and the property 24 .

\section{Noises and regularisation}

A bad conditioning of the Gram covariance matrices, the ratio of their largest and smallest eigenvalues, would prejudice numerically their inversion and transmit more noise from the measurements into the source estimate (Golub and Van Loan, 1983). The use of regularisation techniques, not essential for the algebraic estimate, becomes such when considering the positive one. The iterative removal of parts of the domain $\Omega \times \mathrm{T}$ corresponding to significant contrasts between the retroplumes rapidly degrades the conditioning of the Gram covariance matrix, already at least 300 for $\mathbf{H}_{(2)}$. This section justifies the use of a regularising technique, the truncated singular value decomposition (TSVD) extensively described by Bertero et al. $(1985,1988)$ and already used by Enting et al. (1995) or Fan et al. (1999). It is shown finally that the estimate is sensitive to the square root of the conditioning of the inverted matrices. This explains why so large a conditioning as 60 may be used: only the very small singular values have to be truncated.

The inversion described in the previous two sections, as it works with the retroplumes $r_{i}$ produced by a model, would ideally require synthetic model measurements $\boldsymbol{\mu}^{s}=\boldsymbol{\mu}_{\text {mod }}(\sigma)$. Let's denote the measurements really observed as $\boldsymbol{\mu}^{o}=\boldsymbol{\mu}_{\text {real }}(\sigma)=\boldsymbol{\mu}^{s}+\delta \boldsymbol{\mu}$. The error $\delta \boldsymbol{\mu}=\boldsymbol{\mu}_{\text {real }}(\sigma)-$ $\boldsymbol{\mu}_{\text {mod }}(\sigma)$ gathers errors due to the limited quality of the observations and due to the limited quality of the model. The estimated coefficients $\lambda^{o}$ will correspondingly drift from the ideal values with errors $\delta \lambda$. Suppose that the same measurement was performed twice by two identical detectors operated simultaneously and at the same place. The observations $\mu_{1}^{o}=\mu+\delta \mu_{1}$ and $\mu_{2}^{o}=\mu+\delta \mu_{2}$ correspond to the same ideal measurement $\mu=\mu_{1}=\mu_{2}$ and retroplume $r=r_{1}=r_{2}$. The Eqs. (8) or (19) may now be written as :

$$
\begin{aligned}
& {\left[\begin{array}{l}
\frac{\mu_{1}^{o}+\mu_{2}^{o}}{2} \\
\frac{\mu_{1}^{o}-\mu_{2}^{o}}{2}
\end{array}\right]=\left[\begin{array}{cc}
\|r\|^{2} & 0 \\
0 & 0
\end{array}\right]\left[\begin{array}{c}
\frac{\lambda_{1}^{o}+\lambda_{2}^{o}}{\frac{\lambda_{1}^{o}-\lambda_{2}^{o}}{2}}
\end{array}\right]} \\
& {\left[\begin{array}{c}
\frac{\lambda_{1}^{o}+\lambda_{2}^{o}}{2} \\
\frac{\lambda_{1}^{o}-\lambda_{2}^{o}}{2}
\end{array}\right]=\left[\begin{array}{cc}
\|r\|^{-2} & 0 \\
0 & +\infty
\end{array}\right]\left[\begin{array}{c}
\mu+\frac{\delta \mu_{1}+\delta \mu_{2}}{2} \\
\frac{\delta \mu_{1}-\delta \mu_{2}}{2}
\end{array}\right]}
\end{aligned}
$$

This example shows that the two pieces of information $\mu_{1}^{o}, \mu_{2}^{o}$ may be rearranged and divided into a "relevant" part $\frac{\mu_{1}^{o}+\mu_{2}^{o}}{2}$ and a "redundant" part $\frac{\mu_{1}^{o}-\mu_{2}^{o}}{2}$. The redundant information corresponds to non significant differences between repeated (combinations of) measurements with the risk of an abusive interpretation in terms of the noise. It jeopardises with little eigenvalues the conditioning of the Gram covariance matrix. The presence of redundant information is not totally negative as it reduces the noise touching the relevant information. If the noises in $\mu_{1}^{o}$ and $\mu_{2}^{o}$ are independent with the same standard deviation (the bar denotes the statistical expectation), $m=\sqrt{\overline{\delta \mu_{1}^{2}}}=\sqrt{\overline{\delta \mu_{2}^{2}}}$, the effective noise for the relevant information $\frac{\mu_{1}^{o}+\mu_{2}^{o}}{2}$ has a standard deviation $\frac{m}{2}$. 
In order to improve the quality of the relevant information and to remove the redundant one we regularised by a TSVD the successive Gram matrices here simply denoted $\mathbf{H}$. No tilde, prime symbol or subscript number will be written hereafter. $\mathbf{H}$ is put into diagonal form; as it is positive definite, the transition matrix $\mathbf{O}$ is orthogonal ${ }^{t} \mathbf{O}=\mathbf{O}^{-1}$, the left exponent $t$ stands for the transposition). The eigenvalues written decreasingly display a conditioning $\frac{\theta_{1}}{\theta_{n}}$ that will be reduced to the desired value cond by topping the inversion of the too little ones:

$\mathbf{H}=\mathbf{O}\left[\begin{array}{ccccc}\theta_{1} & & & & \\ & \cdot & & \\ & \theta_{k} & & \\ & & \theta_{k+1} & \\ & & & \cdot \\ & & & & \theta_{n}\end{array}\right] \mathbf{O}^{-1}$

$\theta_{1} \geq . . \geq \theta_{k} \geq \theta_{\text {top }}>\theta_{k+1} \geq . . \geq \theta_{n} \geq 0 \quad \theta_{\text {top }}=\frac{\theta_{1}}{\text { cond }}$

The regularised inverse of $\mathbf{H}$ is taken to be:

$\mathbf{H}^{i n v}=\mathbf{O}\left[\begin{array}{ccccc}\theta_{1}^{-1} & & & & \\ & \cdot & & \\ & \theta_{k}^{-1} & & \\ & & \theta_{t o p}^{-1} & \\ & & & \\ & & & & \\ & & & \theta_{t o p}^{-1}\end{array}\right] \mathbf{O}^{-1}$

Satisfactory results have been obtained in the next sections with such a large conditioning as $c o n d=60$. To see that let's consider the ideal regularised estimate of the source:

$\sigma_{\|}(\boldsymbol{x}, t)=\lambda^{s} \cdot \boldsymbol{r}(\boldsymbol{x}, t) \quad \lambda^{s}=\mathbf{H}^{i n v} \boldsymbol{\mu}^{s}$

When using the observations $\boldsymbol{\mu}^{o}=\boldsymbol{\mu}^{s}+\delta \boldsymbol{\mu}$ the estimation is achieved with some error as:

$\sigma_{\|}^{o}=\sigma_{\|}+\delta \sigma_{\|}=\lambda^{o} \cdot \boldsymbol{r} \quad \lambda^{o}=\mathbf{H}^{i n v} \boldsymbol{\mu}^{o}$

$\delta \sigma_{\|}(\boldsymbol{x}, t)=\delta \boldsymbol{\lambda} \cdot \boldsymbol{r}(\boldsymbol{x}, t) \quad \delta \boldsymbol{\lambda}=\mathbf{H}^{i n v} \delta \boldsymbol{\mu}$

We may evaluate as the trace of a square matrix $\left\|\delta \sigma_{\|}\right\|^{2}={ }^{t} \delta \lambda \mathbf{H} \delta \boldsymbol{\lambda}={ }^{t} \delta \boldsymbol{\mu} \mathbf{H}^{i n v} \delta \boldsymbol{\mu}=\operatorname{tr}\left(\mathbf{H}^{i n v}\left[\delta \boldsymbol{\mu}^{t} \delta \boldsymbol{\mu}\right]\right)$. Hence, if $\mathbf{R}=\overline{\delta \boldsymbol{\mu}^{t} \delta \boldsymbol{\mu}}$ is the statistical covariance matrix of $\delta \mu$ :

$\overline{\left\|\delta \sigma_{\|}\right\|^{2}}=\operatorname{tr}\left(\mathbf{H}^{i n v} \mathbf{R}\right)$

This expression is adapted from (Hunt, 1971). It will be compared to $\left\|\sigma_{\|}^{o}\right\|^{2}=\operatorname{tr}\left(\mathbf{H}^{i n v}\left[\boldsymbol{\mu}^{o t} \boldsymbol{\mu}^{o}\right]\right)$. It strengthens the conclusion that the redundant information, after reducing the effect of the noise, must be removed from the inversion. It shows also that when estimating the standard deviation $\sqrt{\overline{\left\|\delta \sigma_{\|}\right\|^{2}}}$ it is not the conditioning of $\mathbf{H}$ that counts but rather its square root.

In the next sections the effect of the observational and model noises are evaluated directly in the calculations.

\section{Calculations about ETEX1}

On October 1994, between the 23 October, 16:00 UT and the 24 October, 4:00 UT, $340 \mathrm{~kg}$ of an inert tracer, perfluoromethylcyclohexane or pmch, were released from a ground position near the village of Monterfil, Brittany, France. Time averaged concentration measurements were delivered each third hour by 168 detectors all over Europe. The experiment, sponsored by the European Commission together with a second one organised in November the same year (ETEX2), is described in (Joint Res. Centre, 1998). The data are available from the Joint Research Centre, Environment Monitoring Unit, Ispra (Varese), Italy (web site http://java.ei.jrc.it/ etex/database/).

The results presented here have been obtained from two selections of measurements described on Fig. 1. The first selection comprises 48 measurements from 7 stations making up a subnetwork with a characteristic spacing of $300 \mathrm{~km}$. The second one comprises 130 measurements taken by 14 stations with a characteristic spacing of $500 \mathrm{~km}$. The stations have been selected in order to detect the cloud of pmch and its edges. The measurements have been selected for each station in order to capture the arrival, passing and departure of the cloud. In the station of Brest (station number 89 or F8), we could only take into account zero valued measurements containing the information that the cloud had never been there.

In order to investigate the effect of detector and meteorological modelling errors, the source ETEX1 has been rebuilt for different values of the selected measurements:

1. synthetic measurements $\boldsymbol{\mu}^{s}$ obtained from POLAIR

2. synthetic noisy measurements $\boldsymbol{\mu}^{g}$ with $30 \%$ of relative Gaussian noise (see below)

3. real measurements from the ETEX1 database $\boldsymbol{\mu}^{o}=\boldsymbol{\mu}^{s}+\delta \boldsymbol{\mu}$

Note that the hypothesis of an error proportional to the signal is suitable for the observational part, not for the difference between the model and the reality. The $\mu_{i}^{s}$ were transformed into the non negative noisy measurements $\mu_{i}^{g}=\mu_{i}^{s} \max \left(0,1+\alpha_{i}\right)$ by means of two sets of independent random variables $\alpha_{i}, \mathrm{i}=1$ to 48 and $\mathrm{i}=1$ to 130 . The $\alpha_{i}$, Gaussian with $\overline{\alpha_{i}}=0, \sqrt{\overline{\alpha_{i}^{2}}}=30 \%$, were prepared as $\alpha_{i}=0.3 \cos \left(2 \pi x_{i}\right) \sqrt{-2 \log y_{i}}$ using two random independent variables $x_{i}$ and $y_{i}$ equally distributed between 0 and 1. The $x_{i}$ 's and $y_{i}$ 's were obtained from a system supplied law, in fact we suppose a sequence of numbers, having the announced statistical properties. The system works with a "random seed" which means that, if we give the same seed, we obtain the same random sequence $x_{1}, y_{1}, x_{2}, y_{2}, x_{3}, \ldots$ if the seed is changed, another independent sequence is drawn.

In order to really show that the inversion was stable with respect to $30 \%$ of relative noise the calculations should have 

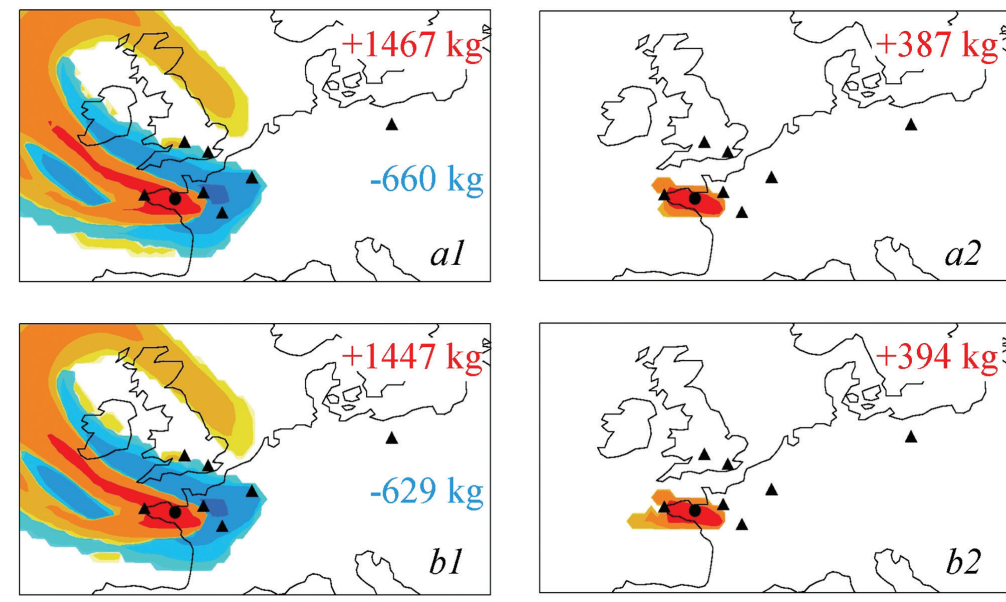

$10^{-4}$
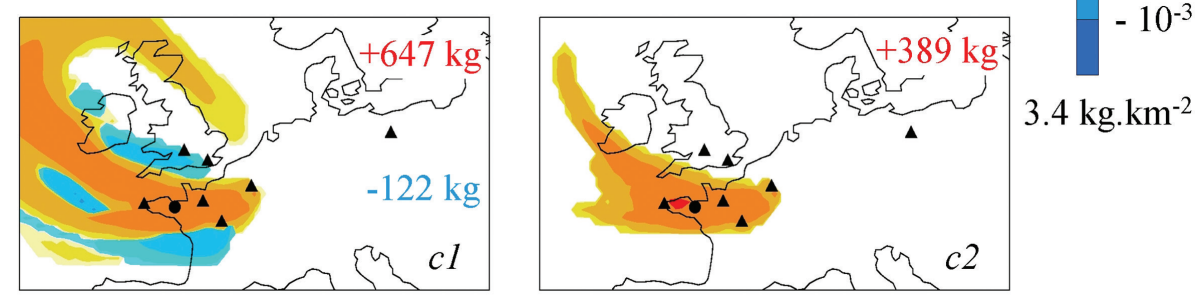

Fig. 4. Results obtained for the selection of 48 measurements when the illumination is taken into account as explained in Sect. 4 . The source is evaluated as a flux at ground or sea level. Monterfil is indicated by a black point and the detectors by triangles. The part (a) of the figure is obtained for synthetic measurements, part (b) for synthetic measurements with $30 \%$ of relative noise, part (c) out of the real measurements from the ETEX1 database. Because of the time integration the positive reconstruction, on the right column, may sometimes be non zero at places where the algebraic reconstruction, on the left column, is negative in apparent contradiction with the proposition 24 . The sum of the positive contributions is indicated in red, in blue for the negative ones.

been repeated with a certain number of seeds. This has been neglected for two reasons. Firstly we considered that, as our random sequence $\alpha_{1}, \alpha_{2}, \ldots$ was not privileged, another random sequence, produced with another seed, would compare qualitatively the same way with the noiseless inversion. Secondly reporting the results would have made the figures bigger and perhaps more difficult to explain.

In order to investigate the potential of the algorithm three artificial sources have been defined and reconstructed with synthetic values for the selection of 130 measurements. These sources correspond to a twelve hour release of $340 \mathrm{~kg}$ of tracer:

1. in southern Belgium, $50^{\circ} 00^{\prime} \mathrm{N}, 4^{\circ} 30^{\prime} \mathrm{E}$, beginning on 23 October 1994, 16:00 UT

2. on the German-Polish boarder, $53^{\circ} 30^{\prime} \mathrm{N}, 14^{\circ} 30^{\prime} \mathrm{E}$, beginning on 25 October 1994, 16:00 UT

3 . on the coast of the Netherlands, $53^{\circ} 00^{\prime} \mathrm{N}, 4^{\circ} 30^{\prime} \mathrm{E}$, beginning on 24 October 1994, 16:00 UT

The adjoint retroplumes were obtained by means of the atmospheric transport model POLAIR (Sportisse et al., 2002; Sartelet et al., 2002; Boutahar et al., 2003) developed at the present Centre d'Enseignement et de Recherche en Environnement Atmosphérique. They have been restricted to the surface (ground or sea level) before going through the inversion. POLAIR is the fruit of a close cooperation with the team in charge at Electricité de France of the passive atmospheric transport model Diffeul (Wendum, 1998). It is a fully modular 3D Eulerian chemistry transport model. Advection is solved with a flux limiter method; diffusion is solved by a three point scheme. The reactive part of the model was switched off for the present application. In order to cover western Europe we used a grid extending from $15^{\circ} \mathrm{W}$ to $35^{\circ} \mathrm{E}$ and from $40^{\circ} \mathrm{N}$ to $67^{\circ} \mathrm{N}$. The horizontal resolution of the model was $0.5^{\circ} \times 0.5^{\circ}$ with fourteen Cartesian levels at $32,150,360, \ldots, 6000 \mathrm{~m}$ above ground or sea level. The calculation went in inverse mode through a period of time starting the 27 October 1994, 7:00 UT (end of the last three hour sampling period) and finishing back in time the 15 October 1994, 00:00 UT. This 296 hour period was covered with a 15 minute time step; concentrations were stored each hour. Meteorological data produced by the European Centre for Mediumrange Weather Forecast were kindly supplied by Météo France. These six-hourly data had the same horizontal resolution as POLAIR but had to be interpolated for the vertical Cartesian levels of the model. Each retoplume required $3 \mathrm{mn}$ CPU time on a PC (Xeon, $1.5 \mathrm{GHz}, 1$ Go RAM).

The calculation of the algebraic and positive surface sources tied to a certain family of measurements was 

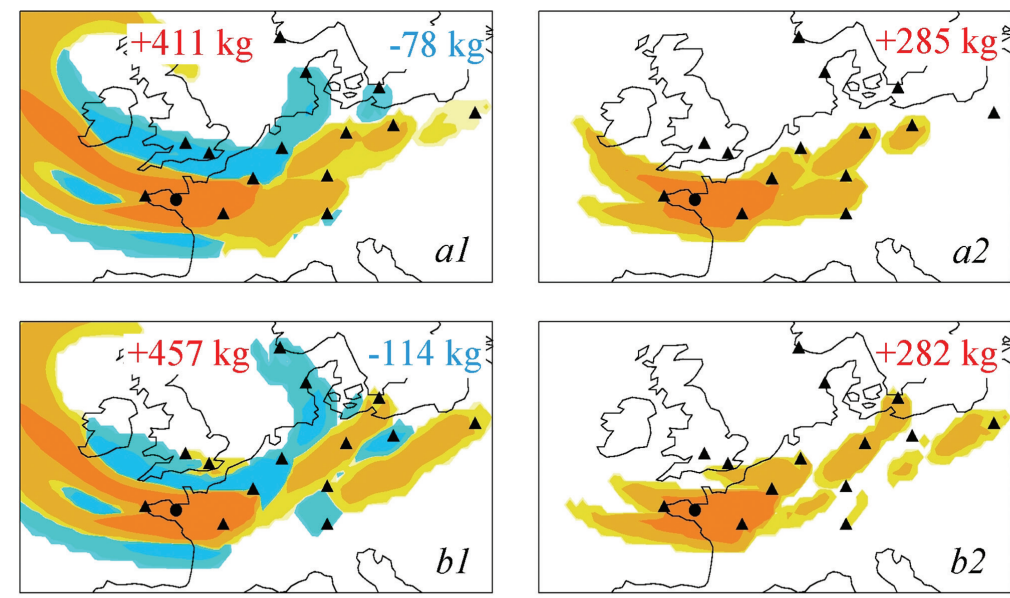

$10^{-3}$

$10^{-4}$
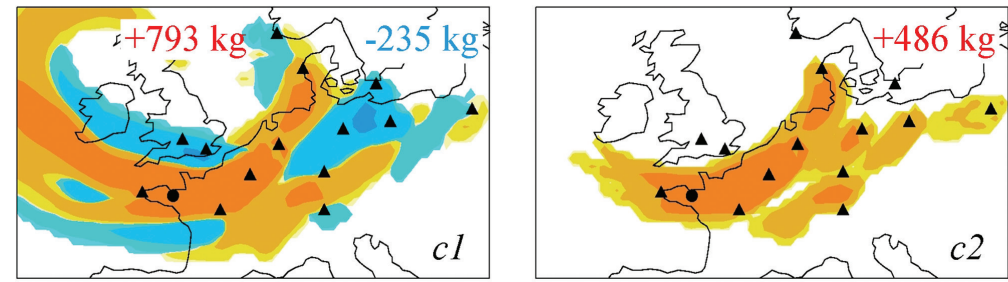

$10^{-5}$

$10^{-6}$

$-10^{-6}$

$-10^{-5}$

$-10^{-4}$

$-10^{-3}$

$3.4 \mathrm{~kg} \cdot \mathrm{km}^{-2}$

Fig. 5. Results obtained for the selection of 130 measurements. The organisation of this figure is parallel to that of Fig. 4 .

organised as follows. A first Gram covariance matrix $\tilde{\mathbf{H}}$ was calculated by visiting each of the $93 * 54 * 296 \simeq 1.510^{6}$ space-time meshes at ground or sea level in order to increment the coefficients $\tilde{h}_{i, j}=\int_{\Omega \times \mathrm{T}} \rho \tilde{r}_{i} \tilde{r}_{j} d \boldsymbol{x} d t$. A first inversion was performed in order to calculate the "éclairement" $\tilde{E}$. The retroplumes were divided by $f=\max \left[\tilde{E}, \frac{\tilde{E}_{\max }}{1000}\right]$. A second Gram covariance matrix $\mathbf{H}_{(1)}$ was calculated out of the smoothed retroplumes leading, through a second inversion, to the algebraic estimate of the source. Successive Gram covariance matrices $\mathbf{H}_{(v)}$ were iteratively obtained, regularised and inverted in order to reach a positive estimate. The number of iterations was generally less than 10 , never more than 20. The conditioning of the first two inversions was always less than the empirical limit cond $=60$, but the successive inversions had to be regularised. The choice $\operatorname{cond}=60$ provides a satisfactory stability of the calculation with respect to the noises as can be seen by comparing Figs. $4 \mathrm{a}$ to $\mathrm{b}$ and $\mathrm{c}$ or $5 \mathrm{a}$ to $\mathrm{b}$ and $\mathrm{c}$. Finally, we controlled the optimality conditions of the positive source. The algebraic estimate restored to within $1 \%$ of error the values of the largest measurements through five orders of magnitude. The positive estimate restored to within $10 \%$ of error the values of the largest measurements through two orders of magnitude. The fact that a measurement is negligible is always restored.

The calculation time for the algebraic source with 48 measurements was $2 \mathrm{mn} \mathrm{CPU}$ on a PC (Xeon, $1.5 \mathrm{GHz}, 1 \mathrm{Go}$ RAM), plus 1 more minute CPU to reach the positive source and control its optimality. With 130 measurements these times become respectively 10 and $5 \mathrm{~min}$. Most of the time is used for calculating the Gram covariance matrices. The regularised inversion of the matrices is very rapid. The calculation time would be roughly multiplied by the number of levels, 14, if the hypothesis of a superficial source was renounced. This hypothesis is made only for its physical relevance and not for numerical convenience.

\section{Results}

The results obtained from the calculations described in the previous section for the estimation of the source ETEX1 may be summarised as follows. The use as base functions of adjoint plumes smoothed according to illumination considerations enables to correct clearly unrealistic features tied to the use of the raw adjoint retroplumes. When it is relevant the positivity constraint clearly improves the estimation. The quality of the reconstruction depends on two things. Firstly, independently of any noise, it depends on the abundance of the data and on the adequacy of the geometry of the monitoring network with respect to the source. Secondly, it depends on the noises and mainly the noise generated by the difference between the model and the reality. The reconstruction is robust when noisy synthetic data are used. Its lesser quality when real data are used is a result at least as much about the ability of the model to fit the reality than about the very inversion. 
An animation would be the best way to view a source $\sigma_{\|}(\boldsymbol{x})$ rebuilt as a function of time at the surface (ground or sea level). As this is not suitable for a paper we decided to cancel the time by showing the time integrated contribution $\Sigma(\boldsymbol{x})$ from all position $x$ at the surface: $\Sigma(\boldsymbol{x})=\int \sigma(\boldsymbol{x}, t) d t$.

As shown by Fig. 2 the source rebuilt by means of raw retroplumes is an unrealistic collection of peaks. This reconstruction amounts to explaining each measurement separately from the others by a local source just a bit spread by the peaked geometry of the corresponding retroplume. The local sources need not be very large and the total amount of tracer released is underestimated. This unphysical reconstruction is also mainly positive, except for little negative compensations as can be seen near the English stations UK7, UK8 on Fig. 2a or b. It follows the geometry of the illumination represented by Fig. $3 a$ and $b$.

When the retroplumes are smoothed the reconstruction becomes more coherent. On Figs. 4a, b, or 5a, b, obtained for synthetic data or Figs. 4c, 5c obtained for real data, the emissions are maximum by the expected position of Monterfil.

The relative uncertainty of the real data is about $15 \%$ (Joint Res. Centre, 1998). This is twice smaller than the $30 \%$ of noise introduced in the synthetic measurements with no significant variation of the algebraic or positive reconstructions as shown by Figs. 4a, b and 5a, b. More significant differences occur for real data on Figs. $4 c$ or 5c, especially in terms of the total positive or negative contributions. Hence, when studying ETEX1, the influence of the observational noise is negligible compared to the influence of model errors. These errors stem in particular from the limited quality of the analysed winds and of the diffusion parameterised according to Louis (1979) and Louis et al. (1982). The line of current departing from the release ETEX1, first flowing eastward progressively bends northward through Germany before passing in Scandinavia. It seems this current line went more east in the model than in the reality. Fig. 6 shows indeed that the concentration of pmch really observed is more at the Danish station DK5, and less at the station DK4, than can be modelled in POLAIR from the source ETEX1. During the inversion of the 130 real measurements, the "excessive" values of the real measurements at DK5 are seen like a local source above the station: compare Figs. 5a and c. Stations D32, DK4 and P16 display an opposite behaviour.

In fact, wind or model noises generate near the spacetime position of the detectors artificial positive or negative releases that are easily identified even if the real source is not known. These extrema provide thus a valuable information about the modelling errors. To a lesser extent in the case of ETEX1 such extrema are also generated by the observational noise. They could also correspond to the reality if the source of tracer is very inhomogeneous and if a detector has been settled close to an important point of release; this explanation is nevertheless not suitable for negative extrema.

The comparison of the results obtained from the two selections is surprising. In order to avoid any interference of

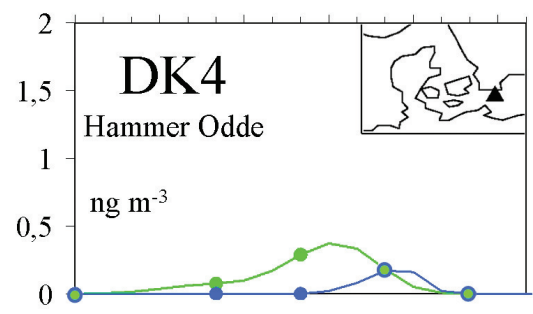

$\mathrm{t}_{0}: 23 / 10 / 94,16 \mathrm{UT}$ real measurements synthetic measurements

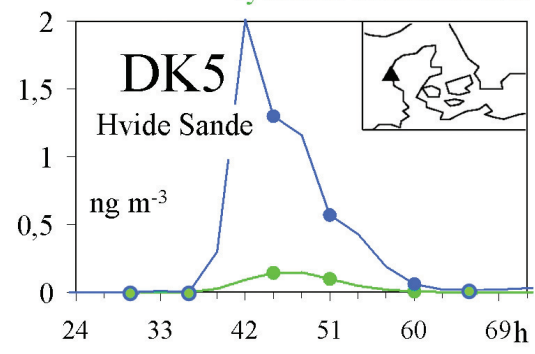

Fig. 6. Evolution of the concentrations observed, in blue, and simulated, in green, at the Danish stations DK4 and DK5 for the sixteen measurements taken three-hourly between the hours 24 to 75 after the beginning of the ETEX1 release. The dots indicate which measurements at these stations have been effectively included in the selection of 130 ones.

the various noises the comparison should be mainly between Figs. 4a and 5a. The algebraic reconstruction out of 48 measurements looks poorer than that out of 130 measurements, but the positive one is much better. We think that the 48 measurement selection provides a better observation of the source ETEX1. This is due to the presence, in this selection only, of measurements taken close to Monterfil by the station F2. The algebraic reconstruction out of 48 measurements turns out poorer but in fact it is more complex because it is tied to a richer information. The quality of the positive reconstructions on Fig. 4a and 5a displays a better agreement with the quality of the data. Our interpretation is that the algorithm is mathematically sound but some physical constraint, a positivity constraint for instance, is apparently required in order to obtain a physically sound result. Positivity is not an acceptable constraint for carbon dioxide. It is generally considered that smoothness is an important feature of this source; such gradients as represented on Fig. 4a would not be acceptable for it. Hence a future evolution of the algorithms described in this paper should be the definition and implementation of some smoothness constraint. This new problem of smoothness of course should not be mistaken for the one addressed in Sect. 4. We must also mention that the source ETEX1 investigated here is all but smooth. It seems that a source varying with typical distances of some hundreds of kilometres would be well seen by such a network as the ETEX subnetworks used in this paper. The resolution would probably benefit from the slowness of the source variations. 
We have noticed that a source was better retrieved when receiving from the detectors a high illumination. As shown by Fig. 3 the illumination $E$ of the source ETEX1 is better with the 48 measurements than with the 130 ones. In order to confirm this additional interpretation of the illumination we evaluated synthetic values for the selection of 130 measurements tied to artificial sources at various positions and dates. Then the sources were reconstructed. Two sources in Belgium and north eastern Germany have been chosen in order to receive the same high illumination a third one was chosen in the Netherlands with a ten times lower value similar to that received by ETEX1. The results reported on Fig. 7 are very explicit. The first two sources are reconstructed with a very good accuracy, the third one is reconstructed with the same accuracy as ETEX1 on Fig. 5a. A first possible explanation would invoke a positive influence of the non smoothed illumination $E$. An alternative explanation can be proposed based on the fact that the illumination smoothed with $f$ given in Eq. (21) is not flat enough so that some regions are still privileged: see Fig. 3a2, b2. This point will have to be further investigated.

\section{The theoretical background}

The inversion strategy exposed in this paper may be placed with respect to the statistical theory of data assimilation formalised by Tarantola (1987). We shall make the comparison with the simplified version of the theory used in most applications in order to show that the ideas developed above could be easily implemented and possibly fruitful. As announced our method is about the choice of the base functions, a point not addressed by the statistical theory. Accordingly our method is compatible and could be combined with the previous developments for, as we shall see, the same numerical price.

In the general theory of the statistical estimation the sought source or state vector $\sigma(\boldsymbol{x}, t)$ is an object with a large dimension determined by the discretisation of the space-time domain. Its dimension may be reduced to the number of available measurements $\mu_{1}, . ., \mu_{n}$ by investigating the estimate $\sigma_{\|}=\sum_{i=1}^{n} \lambda_{i} b_{i}$ as a linear combination of some prescribed base functions $b_{1}, . ., b_{n}$ (we recommend $r_{1}^{\prime}, . ., r_{n}^{\prime}$ ). To obtain the $\lambda_{i}$ 's one calculates the adjoint functions $r_{i}$ (in fact $\tilde{r}_{i}$ ) for each measurement described by the position and date of the receptor (our sampling functions $\pi_{i}$ ). Then, to represent the measurement operator, a matrix $\mathbf{H}$ of elements $h_{i, j}=\left(r_{i}, b_{j}\right)$ is calculated and inverted: $\boldsymbol{\lambda}=\mathbf{H}^{-1} \boldsymbol{\mu}$. A construction of this finite dimensional theory is given by Penenko et al. (2002). The global source of $\mathrm{CO}_{2}$ has been investigated this way by Bousquet et al. (2000); Rödenbeck et al. (2003).

More accurately the method is generally aimed at estimating the mismatch (background error) $\sigma$ between an unknown real source $\sigma_{\text {real }}$ and an a priori estimate $\sigma_{\text {pri }}$ out of the mismatch $\mu$ between the measurements really observed and the values the available model would synthesise for $\sigma_{p r i}$.

$$
\begin{aligned}
\sigma & =\sigma_{\text {real }}-\sigma_{\text {pri }} \\
\boldsymbol{\mu} & =\boldsymbol{\mu}_{\text {real }}\left(\sigma_{\text {real }}\right)-\boldsymbol{\mu}_{\text {mod }}\left(\sigma_{\text {pri }}\right) \\
& =\boldsymbol{\mu}_{\text {mod }}(\sigma)+\left[\boldsymbol{\mu}_{\text {real }}\left(\sigma_{\text {real }}\right)-\boldsymbol{\mu}_{\text {mod }}\left(\sigma_{\text {real }}\right)\right]
\end{aligned}
$$

The residual source $\sigma$ is classically modelled as a realisation of a random process, namely a random source $\sigma_{\epsilon}=\sum_{i=1}^{n} \lambda_{\epsilon, i} b_{i}$, bearing two statistical constraints. These constraints are due on the one hand to the quality of the a priori estimate, on the other hand to the quality of the observations and of the model. Firstly $\sigma_{\epsilon}$, realised as $\sigma$, is expected to be statistically zero with a covariance matrix $\mathbf{B}=\left[\overline{\lambda_{i} \lambda_{j}}\right]$. Secondly the difference or innovation $\boldsymbol{d}\left(\sigma_{\epsilon}\right)=\boldsymbol{\mu}-\boldsymbol{\mu}_{\text {mod }}\left(\sigma_{\text {pri }}+\sigma_{\epsilon}\right)$, realised as $\boldsymbol{\mu}_{\text {real }}\left(\sigma_{\text {real }}\right)-$ $\boldsymbol{\mu}_{\text {mod }}\left(\sigma_{\text {real }}\right)$ is expected to be close to zero with a covariance matrix for observational and modelling errors $\mathbf{R}=$ $\mathbf{R}_{o b s}+\mathbf{R}_{m o d}=\left[\overline{d_{i}\left(\sigma_{\epsilon}\right) d_{j}\left(\sigma_{\epsilon}\right)}\right]$.

The doubly constrained inversion goes through a gain matrix: $\lambda=\left(\mathbf{B}^{-1}+{ }^{t} \mathbf{H} \mathbf{R}^{-1} \mathbf{H}\right)^{-1}{ }^{t} \mathbf{H} \mathbf{R}^{-1} \boldsymbol{\mu}$. This statistical inversion may be formally separated into two parts. Firstly, in order to account for both constraints, the value of the measurements may be corrected as $\boldsymbol{\mu}_{c o r}$. Then, secondly, these measurements are reformulated in terms of a source, which provides the non-statistical core of the inversion :

$$
\begin{aligned}
& \boldsymbol{\mu}_{\text {cor }}=\mathbf{H}\left(\mathbf{B}^{-1}+{ }^{t} \mathbf{H} \mathbf{R}^{-1} \mathbf{H}\right)^{-1}{ }^{t} \mathbf{H} \mathbf{R}^{-1} \boldsymbol{\mu} \\
& \lambda=\mathbf{H}^{-1} \boldsymbol{\mu}_{c o r}
\end{aligned}
$$

In this latter equation the real world acts only on the measurements, $\mathbf{H}^{-1}$ is totally obtained from the model.

Our equations 8, 19 are analogous to this core 42 with, as base functions, the modelled raw or smoothed retroplumes $r_{i}$ or $r_{i}^{\prime}$. Note also that we handle no a priori estimate, which amounts to saying that the a priori estimate is zero $\left(\sigma_{\text {pri }}=0, \sigma=\sigma_{\text {real }}\right)$ and not statistically constraining $\left(\mathbf{B}^{-1}=0, \boldsymbol{\mu}_{\text {cor }}=\boldsymbol{\mu}\right)$.

As announced the main point of our strategy is that the base functions $b_{i}$ are derived from the sensitivity functions $r_{i}$. The availability of the $r_{i}$ represents no additional cost as they are required in other respect. Instead of calculating and inverting one asymmetric matrix $\mathbf{H}=\left[\left(r_{i}, b_{j}\right)\right]$ we calculate and invert for the same cost two symmetric matrices $\mathbf{H}=\left[\left(r_{i}, r_{j}\right)\right]$ and $\mathbf{H}^{\prime}=\left[\left(r_{i}, r_{j}^{\prime}\right)\right]=\left[\left(r_{i}^{\prime}, r_{j}^{\prime}\right)^{\prime}\right]$. Finally the source is estimated as a combination of smoothed $r_{i}^{\prime}$ calculated with an imperfect inverse model rather than as a combination of arbitrarily chosen $b_{i}$.

The statistical approach is generally implemented with a base of smooth functions so that no unrealistic detail is ever deplored. The notion of illumination would be relevant as well in this context, it is more general than the presentation 

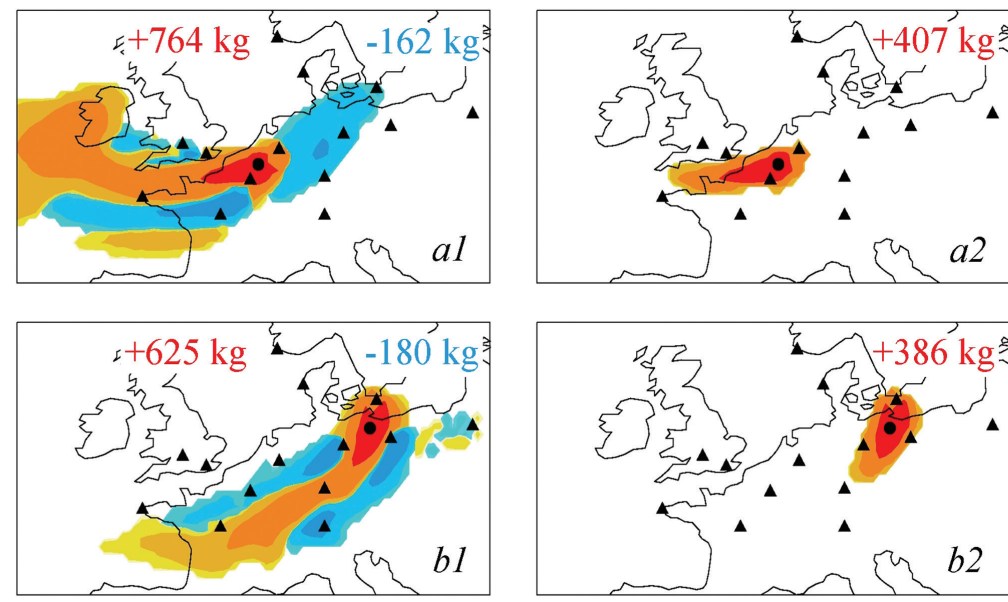

$10^{-4}$

$10^{-5}$

$10^{-6}$
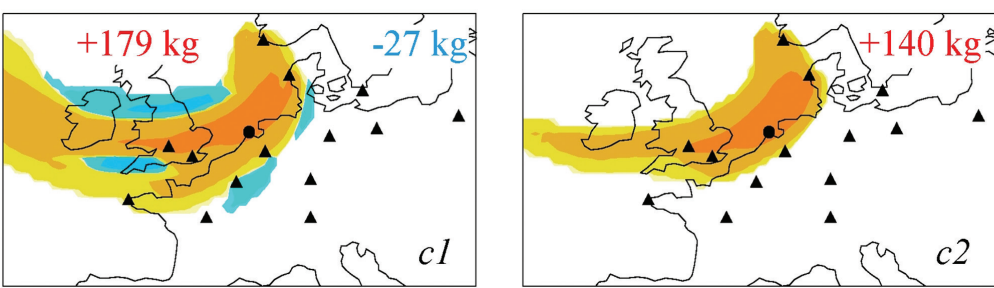

$-10^{-6}$

$-10^{-5}$

$-10^{-4}$

$-10^{-3}$

Fig. 7. Algebraic and positive reconstructions out of synthetic values of the selected 130 measurements for three imaginary sources at positions indicated by the black point: (a) in Belgium, (b) in north-eastern Germany, (c) in the Netherlands. The sources correspond to $340 \mathrm{~kg}$ of tracer released during the twelve hours of best local illumination. As explained for Fig. 4 the results are integrated in time. In (a) and (b) the sources are well illuminated, the reconstruction has a good accuracy, especially in the positive case. In (c) the lesser illumination of the source in the Netherlands is similar to that of the source ETEX1 and so is the lesser quality of its reconstruction.

made in Sect. 4. Suppose we handle some arbitrary $b_{1}, \ldots, b_{n}$. The $b_{i}$ can always be linearly rearranged in such a way that $\mathbf{H}=\left[\left(r_{i}, b_{j}\right)\right]$ is the identity matrix. Then a set of measurements $\boldsymbol{\mu}$ will be interpreted as due to a source $\sigma_{\|}=\sum_{i=1}^{n} \mu_{i} b_{i}$. The point $(\boldsymbol{x}, t)$ will contribute to this source a release $\delta \sigma(\boldsymbol{x}, t)=\sum_{i=1}^{n} \mu_{i} b_{i}(\boldsymbol{x}, t) d \boldsymbol{x} d t$. This elementary release produces an elementary measurement $\delta \boldsymbol{\mu}(\boldsymbol{x}, t)=\mathbf{M}(\boldsymbol{x}, t) \boldsymbol{\mu}$ with a matrix $\mathbf{M}(\boldsymbol{x}, t)=\left[\left(r_{i}(\boldsymbol{x}, t), b_{j}(\boldsymbol{x}, t)\right]\right.$. The matrix $\mathbf{M}(\boldsymbol{x}, t)$ describes the weight attributed to the point $(\boldsymbol{x}, t)$ by the inversion, and this weight may be summarised by introducing $E^{b}(\boldsymbol{x}, t)=\operatorname{tr} \mathbf{M}(\boldsymbol{x}, t)=\sum_{i=1}^{n} r_{i} b_{i}(\boldsymbol{x}, t)$. This definition generalises the formulae 15 and 16 and $E^{b}$ may be regarded as the illumination tied to the base functions $b_{i}$. We think that, in order to have a good interpretation of the measurements, this effective illumination should be as homogeneous as possible otherwise aberrations are expected. For instance, if $E^{b}\left(x_{0}, t_{0}\right)=0$ then one can see that the values at $\left(\boldsymbol{x}_{0}, t_{0}\right)$ of the real and estimated source, $\sigma\left(\boldsymbol{x}_{0}, t_{0}\right)$ and $\sigma_{\|}\left(\boldsymbol{x}_{0}, t_{0}\right)$ are totally independent of one another. Of course we can decide to use an inhomogeneous effective illumination in order to attenuate the importance of regions that are so far from the detectors that the transport processes cannot be modelled reasonably well. The inversion will be based on the influence of the best illuminated regions, and this influence will be propagated to less privileged regions by the shape of the $b_{i}$. Accordingly they should be chosen carefully in order to obtain a relevant propagation of the information.

Any inversion, with arbitrary $b_{i}$, can be formally put in the non smoothed shape of this paper. Nothing is changed in the calculation and use of $\mathbf{H}$ or $\mathbf{H}^{i n v}$ if the sensitivity functions $r_{i}$ are replaced by linear combinations $r_{i}^{b}$ of the $b_{j}$ in such a way that $\left(r_{i}, b_{j}\right)=\left(r_{i}^{b}, b_{j}\right)$ (the $r_{i}^{b}$ are the projections of the $r_{i}$ on the space spanned by the $b_{j}$ ). Exactly in the same way as the $r_{i}$ are the retroplumes associated to the sampling functions $\pi_{i}$, the new functions $r_{i}^{b}$ may be regarded as the retroplumes associated to effective sampling functions $\pi_{i}^{b}=-\frac{\partial r_{i}^{b}}{\partial t}-\boldsymbol{v} \cdot \nabla r_{i}^{b}+\zeta\left(r_{i}^{b}\right)$. Hence, using $b_{i}$ linearly independent of the $r_{i}$ amounts to playing with the definition of the samples. Again this may be justified if we consider that a sample taken at some place and date is in fact representative of a wider area. In particular this is exactly what we do when putting the smoothed $r_{i}^{\prime}$ in the place of the raw $r_{i}$.

\section{Discussion and perspectives}

When retrieving the source of a tracer the choice of the base functions is a pivotal matter. The calculations presented here show that the adjoint concentrations or retroplumes associated to the detectors are not a good choice. The irregularities of these functions are transmitted to the source estimate in the form of unrealistic high resolution details. We 
spontaneously refuse these reconstructions apparently containing, with such details, more information than can be reasonably attributed to the measurements. As an explanation we have introduced a function of illumination. The idea seems fruitful as we have been in a position to smooth the irregularities of the adjoint base functions and to obtain better results. Ongoing investigations by Henry Quiroz (Centro de Modelamiento Matemático, Santiago, Chile) suggest that the technique would even be strictly necessary in some circumstances.

Much work remains to be done in order to strengthen the relevance of the present investigation. The use of the source ETEX1 was very convenient here because it was possible to test the method with real data, because also the source was a space time point emphasising the resolution of the inversion. But our method is firstly aimed at rebuilding diffuse widespread sources. It has to be tested with more realistic geometries; the use of synthetic data will be unfortunately the only possibility.

Theoretical investigations could be open as a consequence of the present inversion strategy with still strong connections with pure and applied mathematics (Ouahsine and Smaoui, 1999; Doubova et al., 2002; Roussel et al., 2002). For instance we noticed in Sect. 4 that the illumination should be homogenised inside some domain of relevance of the monitoring network and attenuated outside. This means we now have to formalise a definition for this domain. It could perhaps be defined in terms of a smoothed illumination $E^{\prime}$ as the region well seen by the smoothed retroplumes. The problem would be to choose the best $E^{\prime}$ and to find the corresponding smoothing function $f$.

In their aforementioned paper Rödenbeck et al. (2003) renounced using $\mathrm{CO} 2$ data from stations that were not operated during the whole period, from 1982 to 2001, in order to avoid that variations in the geometry of the monitoring network might have any influence on their inversion. This influence could be described in terms of the variation of the effective illumination. It could perhaps be reduced by smoothing the base functions in such a way that the effective illumination would be maintained as homogeneous as possible independently of any evolution in the geometry of the monitoring network. Also, we think it could be interesting to investigate their supposed inversion artefact, year 1989 in Africa.

We shall finally remark that the problem of spreading the information away from the detectors is a general care of data assimilation. The theory proposed here is a linear one. The generalisation of automatic differentiation enables to extend data assimilation (Quélo et al., 2002) to a growing number of non linear processes. Non linear extensions of the present work should be addressed as well.

Acknowledgements. The author is deeply grateful to J.-B. Baillon controlling a conjecture, to R. Cominetti explaining the subtlety of optimisation theory under constraints, to A. Osses pointing out possible links with Carleman's control techniques, to L. Gallardo
Klenner and B. Sportisse organising these contacts, to B. Cabrit and to J. Baverel for fruitful past discussions. Graphics have been prepared with the user friendly and public domain graphical package named GrADS originally developed by B. Dotty (COLA, support@grads.iges.org) and maintained with the help of M. Fiorino (LLNL).

\section{References}

Ashbaugh, L. L., Malm, W. C., and Sadeh, W. Z.: A residence time probability analysis of sulfur concentrations at Grand Canyon National park, Atmospheric Environment, 19(8), 1263-1270, 1985.

Baillon, J.-B.: private communication, 25 February 2003.

Baklanov, A.: Numerical modelling for normalisation of atmospheric environment on industrial sites, in: Numerical solution of atmospheric hydrothermodynamics problems, Novosibirsk: Computing Centre RAS, 30-38, 1986.

Baklanov, A.: Modelling of the atmospheric radionuclide transport: local to regional scale, Numerical Mathematics and Mathematical Modelling, INM RAS, Moscow, volume 2, (special issue dedicated to 75-year jubilee of academician G. I. Marchuk), 244-266 (see p. 260 text and figure 5), 2000.

Bertero, M., de Mol, C., and Pike, E. R.: Linear inverse problems with discrete data, I: General formulation and singular system analysis, II: Stability and regularisation, Inverse Problems, 1, 301-330, 1985 (part I) and 4, 573-594, 1988 (part II).

Bousquet, P., Peylin, P., Ciais, P., Rayner, P., Friedlingstein, P., Lequere, C., and Tans, P.: Interannual $\mathrm{CO}_{2}$ sources and sinks as deduced by inversion of atmospheric $\mathrm{CO}_{2}$ data, Science, 290, 1342-1346, 2000.

Boutahar, J., Lacour, S., Mallet, V., Quélo, D., Roustan, Y. , and Sportisse, B.: Development and validation of a fully modular platform for the numerical modeling of air pollution: POLAIR, accepted for publication in International Journal of Environment and Pollution, 2003.

Brandt, J., Ebel, A., Elbern, H., Jakobs, H., Memmesheimer, M., Mikkelsen, T., Thykier-Nielsen, S., and Zlatev, Z.: The importance of accurate meteorological input fields and accurate planetary boundary layer parameterizations, tested against ETEX1, in ETEX symposium on long-range atmospheric transport, model verification and emergency response, 13-16 May 1997, Vienna, Austria, Nodop editor, Proceedings, European Commission, EUR 17346 EN, 195-198, 1997.

Cheney, E. W.: Introduction to approximation theory, International series in pure and applied mathematics, McGraw-Hill, 1966.

Doubova, A., Osses, A., Puel, J.-P.: Exact controllability to trajectories for semilinear heat equations with discontinuous diffusion coefficients. A tribute to J. L. Lions., ESAIM Control Optim. Calc. Var. 8, 621-661, 2002.

de Villiers, G. D., McNally, B., and Pike, E. R.: Positive solutions to linear inverse problems, Inverse Problems, 15, 615-635, 1999.

Enting, I. G., Trudinger C. M., and Francey, R. J.: A synthesis inversion of the concentration and $\delta^{13} \mathrm{C}$ of atmospheric $\mathrm{CO}_{2}$, Tellus, 47B, 35-52, 1995.

Enting, I.G.: Green's function methods of tracer inversion, in Kasibhatla, P., Heimann, M., Rayner, P., Mahowald, N., Prinn, R.G. and Hartley, D.E. editors, Inverse methods in global biogeochem- 
ical cycles, Geophysical Monograph Series, American Geophysical Union, Washington, DC, 2000.

Fan, S. M., Sarmiento, J. L., Gloor, M., and Pacala, S. W.: On the use of regularization techniques in the inverse modeling of atmospheric carbon dioxide, JGR, Vol. 104, 17, 21 503-21 512, 1999.

Gallardo, L., Olivares, G., Langner, J., and Aarhus, B.: Coastal lows and sulfur air pollution in central Chile, Atmospheric Environment, 36, 3829-3841, 2002.

Golub, G. H. and Van Loan, C. F.: Matrix computations, The Johns Hopkins University Press, Baltimore, 1983.

Gram, J. P.: Om Rackkendvilklinger bestemte ved Hjaelp af de minfste Kvadraters Methode, Copenhagen, 1879, translated in German as: Über die Entwicklung reeller Funktionen in Reihen mittels der Methode der kleinsten Quadrate, Journal für reihe und angewandte Mathematik, 94, 41-73, 1883.

Hourdin, F. and Issartel, J.-P.: Subsurface nuclear tests monitoring through the CTBT xenon network, Geophysical Research Letters, 27(15), 2245-2248, 2000.

Hunt, B. R.: Biased estimation for non parametric identification of linear systems, Mathematical Biosciences, 10, 215-237, 1971.

Hürwitz, H.J.: A note on the theory of danger coefficient, Knolls Atomic Power Laboratory report series 48 (KAPL48), 1948.

Issartel, J.-P. and Baverel, J.: Inverse transport for the verification of the CTBT, Atmos. Chem. Phys., 3, 475-486, 2003.

Joint Research Centre: Etex. The European tracer experiment, European communities, EUR 18143 EN, ISBN 92-828-5007-2, 107, 1998

Lewins, J.: Importance, the Adjoint Function, Pergamon Press, 172 P. (see especially p. 20-28), 1965.

Louis, J.-F.: A parametric model of vertical eddy fluxes in the atmosphere, Boundary Layer Meteorology, 17, 187-202, 1979.

Louis, J.-F., Tiedke, M., and Geleyn, J.-F.: A short history of the PBL parameterization at ECMWF, Proceedings ECMWF workshop on planetary boundary layer parameterization, 59-79, 1982.

Marchuk, G. I.: Sopryazhennye Uravneniya i Analiz Slozhnykh Sistem, Moskva, Nauka, 1992, translated in English as: Adjoint Equations and Analysis of Complex Systems, Mathematics and its Applications, Vol. 295, Hazewinkel editor, Kluwer Academic Publisher, 1995.

Marchuk, G. I.: Perturbation theory and the statement of inverse problems, Lecture Notes in Computer Science, Vol. 4, 159, 1973.

Marchuk, G. I.: Equation for value of information from meteorological satellites and formulation of inverse problems, Space Research, Vol. 2, No. 3, 462-477, 1964.

Ouahsine, A. and Smaoui, H.: Flux-limiter schemes for oceanic tracers: application to the English Channel tidal model, Comput. Methods Appl. Mech. Engrg. 179, 307-325, 1999.

Penenko, V. and Baklanov, A.: Methods of sensitivity theory and inverse modeling for estimation of source term and nuclear risk/vulnerability areas, Lecture Notes in Computer Science (LNCS), Springer, 2074, 57-66, 2001.

Penenko, V., Baklanov, A., and Tsvetova, E.: Methods of sensitivity theory and inverse modeling for estimation of source term, Future Generation Computer Systems, 18, 661-671, 2002.

Pudykiewicz, J.: Application of adjoint tracer transport equations for evaluating source parameters, Atmospheric Environment, 32(17), 3039-3050, 1998.

Quĺo, D., Sportisse, B., Berroir, J.-P., Charpentier, I.: Some remarks concerning inverse modeling and data assimilation for slow-fast atmospheric chemical kinetics, in Sportisse editor, proceedings of the second conference on air pollution modelling and simulation, organised by ENPC and INRIA, Champs-sur-Marne, 9-12 April, Springer, 499-513, 2001.

Rockafellar, R. T.: Convex analysis, Princeton University Press, 1970.

Rödenbeck, C., Houweling, S., Gloor, M., and Heimann, M.: $\mathrm{CO}_{2}$ flux history 1982-2001 inferred from atmospheric data using a global inversion of atmospheric transport, Atmos. Chem. Phys. Disc., 5 November 2003.

Roussel, G., Ternsisien, E., and Benjelloun, M.: Estimation d'un modle stationnaire de dispersion et localisation de source. Application la surveillance de la pollution, Revue du Traitement du Signal, 19(1), 37-48, 2002.

Sartelet, K. N., Boutahar, J., Quélo, D., Coll, I., and Sportisse, B.: Development and validation of a 3D chemistry transport model POLAIR3D, by comparison with data from ESQUIF campaign, Proc. of the 6th GLOREAM workshop: Global and Regional Atmospheric Modelling, Aveiro, Portugal, 4-6 September, 2002.

Seibert, P.: Inverse modelling with a Lagrangian particle dispersion model: application to point releases over limited time intervals, in Gryning, S.E. and Schiermeier, F.A. editors, Air Pollution and its Application XIV, 381-389, NATO, Kluwer Academic/Plenum Publisher, 2001.

Seinfeld, J. H. and Pandis, S. N.: Atmospheric chemistry and physics, Wiley-Interscience Publication, 1998.

Sharan, M., McNider, R. T., Gopalakrishnan, S. G., and Singh, M. P.: Bhopal gas leak: a numerical simulation of episodic dispersion, Atmospheric Environment, 29, 2051-2059, 1995.

Smith, F. B.: The diffusion of smoke from a continuous elevated point-source into a turbulent atmosphere, J. Fluid Mech., 2, 4976 (see p. 66), 1956.

Soodak, H.: The science and engineering of nuclear power, United Nations, New York, 1948.

Sportisse, B., Boutahar, J., Debry, E., Quélo, D., and Sartelet, K.: Some tracks in air pollution modeling and simulation, Revista de la Real Academia de Ciencias, Serie A: Matemáticas (RACSAM), 96(3), 507-528, 2002.

Stohl, A.: Computation, accuracy and applications of trajectories - a review and bibliography, Atmospheric Environment, 32(6), 947-966 (see p. 959), 1998.

Tarantola, A.: Inverse problem theory, Elsevier, 1987.

Uliasz, M. and Pielke, R.: Application of the receptor oriented approach in mesoscale dispersion modeling, in van Dop, $\mathrm{H}$. and Steyn, D.G. editors, Air Pollution and its Application VIII, 399407, NATO, Plenum Publisher, 1991.

Weinberg, A. M. and Wigner, E. P.: The physical theory of neutron chain reactors, University Press, Chicago, 1958.

Wendum, D.: Three long-range transport models compared to the ETEX experiment: a performance study, Atmospheric Environment, 32(24), 4297-4305, 1998.

Wigner, E.P.: Effects of small perturbations on pile period, Chicago Report P-G, 3048, 1945.

Wotawa, G., de Geer, L.-E., Denier, P., Kalinowski, M., Toivonen, H., d'Amours, R., Desiato, F., Issartel, J.-P., Langer, M., Seibert, P., Frank, A., Sloan, C., and Yamazawa, H.: Atmospheric transport modelling in support of CTBT verification - overview and basic concepts, Atmospheric Environment 37, 2525-2537, 2003. 